\title{
Fatores associados às velocidades de ganho de peso e de comprimento nos primeiros seis meses de vida
}

\author{
Factors associated with length and weight gain rates \\ during the first six months of life
}

\author{
Sarah Aparecida Vieira1, Taís Cristina Araújo Magalhães², \\ Andréia Queiroz Ribeiro ${ }^{3}$, Silvia Eloiza Priore ${ }^{4}$, \\ Sylvia do Carmo Castro Franceschini ${ }^{4}$, Luciana Ferreira da Rocha Sant'Ana ${ }^{3}$
}

\begin{abstract}
Resumo
O estudo teve como objetivo verificar os fatores associados às velocidades de ganho de peso (VGP) e de comprimento (VGC) de lactentes nos primeiros quatro a seis meses de vida. Estudo transversal, com análise de dados secundários, no qual foram obtidas informações em prontuários de atendimento de 371 lactentes acompanhados por um Programa de Apoio à Lactação. Foram calculadas as VGP e VGC em lactentes do nascimento aos quatro a seis meses de vida. Foram investigados os fatores associados às VGP e VGC por meio de regressão linear múltipla. Verificou-se que os lactentes do sexo feminino e os que não estavam em aleitamento materno exclusivo ou predominante apresentaram menor VGP aos quatro a seis meses. Os lactentes cujas mães não viviam com companheiro, os filhos de mães que trabalhavam fora do lar e as que realizaram menos de seis consultas no pré-natal apresentaram menor VGC. Lactentes com maior comprimento ao nascer também apresentaram menor VGC aos quatro a seis meses. Os resultados deste estudo evidenciam a diversidade de fatores que podem estar associados às VGP e VGC nos primeiros meses de vida, como a prática do aleitamento materno, a atenção pré-natal e as variáveis socioeconômicas. A identificação precoce dos fatores relacionados ao crescimento de lactentes pode direcionar ações para promoção do adequado desenvolvimento nessa fase.
\end{abstract}

Palavras-chave: lactente; ganho de peso; crescimento; associação.

\begin{abstract}
The study aimed to verify factors associated with weight (WGR) and length (LGR) gain rates of infants during their first 4 to 6 months of life. A cross-sectional study with secondary data analysis, that collected information on medical charts of 371 infants followed during their first six months of life by the Lactation Support Program. The WGR and length LGR gain of infants from birth to 4-6 months old were calculated. Factors associated with WGR and LGR were examined by multiple linear regression analysis. We found that female infants and those that were not exclusively or predominantly breastfed had lower WGR at 4-6 months. Infants whose mothers were not living with a partner, infants of working mothers, and mothers that had less than six prenatal visits during their pregnancy had a lower LGR. Infants with a greater birth length also had a lower LGR at 4-6 months. The results of this study highlight the diversity of factors the may be associated with the length and weight gain rate during the first months of life, such as breastfeeding, prenatal care and socioeconomic variables. The early identification of factors related to infants growth can direct actions promote a proper development on that stage.
\end{abstract}

Keywords: infant; weight gain; growth; association.

\footnotetext{
1 Doutoranda em Ciência da Nutrição na Universidade Federal de Viçosa (UFV) - Viçosa (MG), Brasil.

${ }^{2}$ Mestre em Ciência da Nutrição na Universidade Federal de Viçosa (UFV) - Viçosa (MG), Brasil.

${ }^{3}$ Professora adjunta do Departamento de Nutrição e Saúde na Universidade Federal de Viçosa (UFV) - Viçosa (MG), Brasil.

${ }^{4}$ Professora associada do Departamento de Nutrição e Saúde na Universidade Federal de Viçosa (UFV) - Viçosa (MG), Brasil.

Trabalho realizado na Universidade Federal de Viçosa (UFV) - Viçosa (MG), Brasil.

Endereço para correspondência: Sarah Aparecida Vieira - Departamento de Nutrição e Saúde, Edifício Centro de Ciências Biológias II, Campus Universitário, s/n - CEP: 3670-900 - Viçosa (MG), Brasil - Email: sarahvieiraufv@gmail.com

Fonte de financiamento: Fundação de Amparo à Pesquisa do Estado de Minas Gerais - Fapemig. Número do processo: Fapemig 02055-13. Conselho Nacional de Desenvolvimento Científico e Tecnológico - CNPq. Número do processo: CNPQ 485124/2011-4.
}

Conflito de interesses: nada a declarar. 


\section{INTRODUÇÃO}

Nos primeiros meses de vida, o crescimento é caracterizado como um processo complexo e com mudanças rápidas, que é afetado direta ou indiretamente por fatores como sexo, condições de nascimento, tipo de alimentação e condições de saúde ${ }^{1,2}$.

O estudo do crescimento tem contribuído para o acompanhamento clínico das crianças e auxiliado no diagnóstico de situações passíveis de prevenção. Há grande interesse em investigar os fatores determinantes do crescimento nos primeiros meses de vida, principalmente devido ao impacto das alterações desse processo em médio e longo prazos, como atraso do desenvolvimento neuropsicomotor e aumento do risco de morbimortalidade ${ }^{3,4}$.

Além dos indicadores de estado nutricional, por exemplo, peso, comprimento e Índice de Massa Corporal, o crescimento pode também ser avaliado pelo cálculo de sua velocidade, expressa pelo ganho de peso ou de comprimento em um intervalo de tempo determinado. No acompanhamento do estado nutricional da criança, o cálculo da velocidade de crescimento tem sido de grande importância, pois, como uma única medida de peso ou de comprimento não é suficiente para diagnóstico nutricional, aponta melhor a ocorrência de problemas ${ }^{2,5}$.

Nos países em desenvolvimento como o Brasil, é observada redução da velocidade de crescimento no primeiro semestre de vida da criança ${ }^{3}$. Acredita-se que tal redução seja justificada pelo fato de ocorrer, nessa fase, a substituição do leite materno por alimentos de baixo valor nutricional e, muitas vezes, contaminados, com consequente aumento da vulnerabilidade a infecções, principalmente as doenças diarreicas ${ }^{3}$. Além disso, a maioria dessas crianças pertence a famílias com condições socioeconômicas precárias, o que as tornam ainda mais vulneráveis ao acometimento por essas doenças ${ }^{6}$. O primeiro ano de vida corresponde a uma época de maior vulnerabilidade a agravos nutricionais, pois compreende um período de intenso desenvolvimento. Assim, é de grande importância o monitoramento do crescimento nessa fase $\mathrm{e}^{7}$.

Com base na comprovada natureza multicausal do crescimento infantil, estudos têm sido desenvolvidos buscando relacionar variáveis biológicas, socioeconômicas, ambientais, culturais, demográficas, entre outras, com a sua etiologia e desenvolvimento ${ }^{1,2}$. Diante do exposto, este estudo teve como objetivo verificar os fatores associados às velocidades de ganho de peso (VGP) e de comprimento (VGC) de lactentes nas idades entre quatro a seis meses atendidos por um Programa de Apoio à Lactação (PROLAC) do município de Viçosa, em Minas Gerais.

\section{MÉTODOS}

Trata-se de um estudo transversal analítico com análise de dados secundários, no qual foram obtidas informações de prontuários de atendimento de lactentes acompanhados nos primeiros seis meses de vida pelo PROLAC no município de Viçosa/MG.

O PROLAC é um Programa de Extensão da Universidade Federal de Viçosa, em parceria com o Banco de Leite Humano de Viçosa/MG, e tem como principais atividades a orientação às mães no período pós-parto, com vistas à promoção do aleitamento materno e ao atendimento nutricional a nutrizes e crianças no seu primeiro ano de vida. Todas as nutrizes do município interessadas em realizar o acompanhamento do bebê no primeiro ano de vida são atendidas pelo Programa.

A amostra do estudo foi composta por prontuários de lactentes que iniciaram o acompanhamento no PROLAC entre agosto de 2003 (data de início do Programa) a novembro de 2007 ( $\mathrm{n}=797$ ) e satisfaziam aos seguintes critérios de inclusão: ter registro do peso e de comprimento ao nascer, peso e comprimento com idades entre quatro a seis meses de vida, ter nascido a termo (entre 37 e 41 semanas de gestação) e não ter nascido com baixo peso ou macrossomia (peso ao nascer $\geq 2.500 \mathrm{~g} \mathrm{e}<4.000 \mathrm{~g}$ ). Como critério de exclusão, considerou-se a presença de alterações de saúde, informadas em prontuário, que pudessem influenciar no estado nutricional nos primeiros meses de vida, como problemas neurológicos e doenças congênitas (três pacientes). Após a seleção dos prontuários com base nos critérios de inclusão e exclusão, foram analisados dados de 371 crianças.

No primeiro atendimento dos lactentes no Programa, foram obtidas informações de peso e de comprimento ao nascer, idade gestacional e tipo de parto, a partir de dados da caderneta de saúde da criança, obtidos do Ministério da Saúde e registrados no prontuário. Foi solicitado às nutrizes que levassem para o primeiro atendimento o cartão de acompanhamento pré-natal para obtenção de informações gestacionais.

A coleta de dados nos prontuários de atendimento do PROLAC ocorreu no período entre julho e outubro de 2010, realizada por duas nutricionistas treinadas e com experiência de atuação no Programa, a fim de evitar erros na interpretação dos dados registrados nos prontuários.

Para cálculo das VGP e de VGC das crianças, foram considerados os dados de peso e de comprimento aos seis meses de vida. Nos casos daquelas crianças que não foram avaliadas nessa idade, consideraram-se as medidas obtidas nas idades de quatro ou cinco meses. Para aferição do peso, foi utilizada balança pediátrica com capacidade de $25 \mathrm{~kg}$ e sensibilidade de 5 g. Para o comprimento, utilizou-se antropômetro infantil com extensão de $1 \mathrm{~m}$, dividido em centímetros e subdivido em milímetros. Para obtenção dos dados antropométricos, a cada consulta foram seguidas técnicas padronizadas ${ }^{8}$.

Para o cálculo da VGP, foi utilizada a Fórmula 1; para o VGC, a Fórmula 2:

$\operatorname{VGP}($ gramas $/$ dia $)=\left(\begin{array}{l}\text { peso aos } 4-6 \text { meses }- \\ \text { peso ao nascer }\end{array}\right) \div$ idade em dias 
VGC (centímetros / mês $)=\left(\begin{array}{l}\text { comprimento aos } 4- \\ 6 \text { meses }- \\ \text { comprimento ao nascer }\end{array}\right) \div$ idade em meses

Para caracterizar o estado nutricional dos lactentes aos quatro a seis meses foram considerados os índices antropométricos Peso/Idade (P/I), Comprimento/Idade (C/I) e Índice de Massa Corporal/Idade (IMC/I), obtidos com o Software WHO Anthro Plus da Organização Mundial da Saúde (OMS). O diagnóstico do estado nutricional das crianças foi realizado seguindo a recomendação em escore-z da OMS 9 .

Foram obtidos nos prontuários de atendimento dados sobre variáveis maternas, gestacionais, de nascimento, prática de aleitamento materno e sociodemográficas, que podem influenciar a VGP e a VGC nos primeiros meses de vida ${ }^{2,4}$. Para determinar o tipo de aleitamento materno (exclusivo ou predominante), adotou-se a classificação da $\mathrm{OMS}^{10}$.

Os dados foram digitados e armazenados em um banco elaborado no Microsoft Office Excel 2007 e as análises foram conduzidas nos programas Social Package Statistical Science (SPSS) for Windows, versão 11.0, e STATA, versão 9.1 (análises de regressão linear). Foi aplicado o teste de Shapiro-Wilk para verificar a normalidades dos dados. Para comparação dos grupos, foram utilizados os testes não paramétricos de Mann-Whitney e Kruskal-Wallis e os testes paramétricos t de Student e Análise de Variância One Way (ANOVA). Para verificar a correlação entre variáveis contínuas, aplicaram-se os testes de Correlação de Pearson e de Correlação de Spearman. A análise de regressão linear múltipla foi realizada para avaliar o efeito das variáveis independentes sobre a VGP e a VGC nos primeiros meses de vida. $\mathrm{O}$ critério definido para inclusão das variáveis na regressão linear múltipla foi a relação com a variável dependente na análise de regressão linear simples, considerando um valor de $\mathrm{p}<0,20$. Para avaliar a adequação e o ajuste do modelo de regressão linear, testou-se a normalidade da distribuição dos resíduos, e a presença de heterocedasticidade foi verificada pelo método de White. Como indicador de multicolinearidade, utilizou-se o fator de inflação da variância. A variável dependente VGP não apresentou distribuição normal e foi submetida à transformação logarítmica para inclusão nas análises de regressão linear. O nível de significância estatística adotado foi $\alpha=5 \%$.

O estudo foi aprovado pelo Comitê de Ética em Pesquisa com Seres Humanos da Universidade Federal de Viçosa (Of. Ref. No 094/2011).

\section{RESULTADOS}

Do total de 371 lactentes, $186(50,1 \%)$ eram do sexo masculino. Entre os lactentes avaliados, 3,5\% apresentaram baixo peso, $2,4 \%$, peso elevado, e 3,7\%, baixo comprimento para idade. Foi também constatado que $2,4 \%$ dos lactentes apresentaram magreza, e 15,0\%, excesso de peso, de acordo com o IMC/I. Nos intervalos de idades estudados (0-4 meses, 0-5 meses e 0-6 meses), os lactentes do sexo masculino apresentaram maior mediana de ganho de peso e maior média de ganho de comprimento em comparação às do sexo feminino $(\mathrm{p}<0,001)$.

A Tabela 1 mostra as características da amostra e a comparação de medianas e médias de VGP e de VGC, de acordo com as

Tabela 1. Mediana (mínimo-máximo), média e desvio-padrão para velocidades de ganho de peso e de comprimento nos primeiros meses de vida de crianças acompanhadas no PROLAC, segundo variáveis estudadas, Viçosa/MG, 2012

\begin{tabular}{|c|c|c|c|c|c|}
\hline \multirow[b]{2}{*}{ Variáveis } & \multirow[b]{2}{*}{ n (\%) } & \multicolumn{2}{|c|}{ VGP } & \multicolumn{2}{|c|}{ VGC } \\
\hline & & $\begin{array}{c}\text { Mediana } \\
\text { (Mín-Máx) (g/dia) }\end{array}$ & Valor p & $\begin{array}{c}\overline{\mathrm{X}}(\mathrm{DP}) \\
(\mathrm{cm} / \mathrm{mês})\end{array}$ & Valor $\mathbf{p}$ \\
\hline Sexo & & & $0,01^{\star}$ & & $0,64^{\ddagger}$ \\
\hline Masculino & $186(50,1)$ & $24,6(10,7-42,9)$ & & $2,8(0,36)$ & \\
\hline Feminino & $185(49,9)$ & $23,6(7,3-51,6)$ & & $2,8(0,40)$ & \\
\hline Estado civil materno & & & $0,07^{\star}$ & & $<0,001^{\ddagger}$ \\
\hline Sem companheiro & $101(27,2)$ & $23,4(14,5-37,9)$ & & $2,7(0,30)$ & \\
\hline Com companheiro & $270(72,8)$ & $24,9(7,3-51,6)$ & & $2,8(0,40)$ & \\
\hline Trabalho materno & & & $0,63^{*}$ & & $0,07^{\ddagger}$ \\
\hline Sim & $150(40,4)$ & $23,9(12,2-37,3)$ & & $2,8(0,36)$ & \\
\hline Não & $221(59,6)$ & $24,2(7,3-51,6)$ & & $2,8(0,39)$ & \\
\hline Escolaridade materna $^{1}$ & & & $0,14^{\star}$ & & $0,52^{\ddagger}$ \\
\hline$\leq 8$ anos & $154(41,7)$ & $23,9(14,1-51,6)$ & & $2,8(0,37)$ & \\
\hline$>8$ anos & $215(58,3)$ & $24,5(7,3-49,5)$ & & $2,8(0,39)$ & \\
\hline Escolaridade paterna $^{2}$ & & & $0,15^{\star}$ & & $0,99^{\ddagger}$ \\
\hline$\leq 8$ anos & $176(51,3)$ & $23,5(14,1-51,6)$ & & $2,8(0,39)$ & \\
\hline$>8$ anos & $167(48,7)$ & $24,4(7,3-40,3)$ & & $2,8(0,37)$ & \\
\hline
\end{tabular}

VGP: velocidade de ganho de peso; VGC: velocidade de ganho de comprimento; AME: aleitamento materno exclusivo; Mín: mínimo; Máx: máximo; $\overline{\mathrm{X}}$ : média; DP: desvio-padrão. ${ }^{*}$ teste de Mann-Whitney. ${ }^{\dagger}$ teste de Kruskal Wallis. ${ }^{\text {t }}$ teste t de Student $;{ }^{5}$ ANOVA. ${ }^{1} \mathrm{n}=369 .{ }^{2} \mathrm{n}=343 .{ }^{3} \mathrm{n}=345 .{ }^{4} \mathrm{n}=343 .{ }^{5} \mathrm{n}=356 .{ }^{6} \mathrm{n}=365 .{ }^{7} \mathrm{n}=362$. ${ }^{8} \mathrm{n}=342 .{ }^{9} \mathrm{n}=370$ 
Tabela 1. Continuação...

\begin{tabular}{|c|c|c|c|c|c|}
\hline \multirow[b]{2}{*}{ Variáveis } & \multirow[b]{2}{*}{ n (\%) } & \multicolumn{2}{|c|}{ VGP } & \multicolumn{2}{|c|}{ VGC } \\
\hline & & $\begin{array}{c}\text { Mediana } \\
(\text { Mín-Máx) (g/dia) }\end{array}$ & Valor $\mathbf{p}$ & $\begin{array}{c}\bar{X}(\mathrm{DP}) \\
(\mathrm{cm} / \mathrm{mês})\end{array}$ & Valor $\mathbf{p}$ \\
\hline Renda familiar ${ }^{3}$ & & & $0,57^{\dagger}$ & & $0,39^{\S}$ \\
\hline$\leq 1$ salário mínimo & $138(40,0)$ & $23,7(7,3-51,6)$ & & $2,8(0,39)$ & \\
\hline 2-4 salários míninos & $179(51,9)$ & $24,4(12,2-42,9)$ & & $2,8(0,38)$ & \\
\hline$\geq 5$ salários mínimos & $28(8,1)$ & $23,9(16,7-34,3)$ & & $2,7(0,39)$ & \\
\hline Número de filhos & & & $0,18^{\star}$ & & $0,95^{\ddagger}$ \\
\hline 1 & $230(62,0)$ & $24,5(7,3-51,6)$ & & $2,8(0,38)$ & \\
\hline$>1$ & $141(38,0)$ & $23,8(12,2-42,3)$ & & $2,8(0,37)$ & \\
\hline Início do pré-natal ${ }^{4}$ & & & $0,87^{\star}$ & & $0,50^{\ddagger}$ \\
\hline$\leq 3$ meses de gestação & $257(74,9)$ & $24,0(7,3-51,6)$ & & $2,8(0,39)$ & \\
\hline$>3$ meses de gestação & $86(25,1)$ & $24,0(14,4-39,3)$ & & $2,8(0,38)$ & \\
\hline $\mathrm{N}^{0}$ de consultas pré-natal ${ }^{5}$ & & & $0,34^{*}$ & & $0,01^{*}$ \\
\hline$<6$ & $143(40,2)$ & $23,7(14,1-51,6)$ & & $2,7(0,37)$ & \\
\hline$\geq 6$ & $213(59,8)$ & $24,0(7,3-49,5)$ & & $2,8(0,38)$ & \\
\hline Tabagismo $^{6}$ & & & $0,13^{\star}$ & & $0,53^{\ddagger}$ \\
\hline Sim & $24(6,6)$ & $24,0(14,1-29,9)$ & & $2,8(0,38)$ & \\
\hline Não & $341(93,4)$ & $24,0(7,3-51,6)$ & & $2,8(0,38)$ & \\
\hline Consumo de álcool ${ }^{7}$ & & & $0,27^{\star}$ & & $0,64^{\ddagger}$ \\
\hline Sim & $21(5,8)$ & $24,5(16,7-49,5)$ & & $2,8(0,36)$ & \\
\hline Não & $341(94,2)$ & $23,9(7,3-51,6)$ & & $2,8(0,38)$ & \\
\hline Ganho de peso gestacional ${ }^{8[11]}$ & & & $0,61^{\dagger}$ & & $0,88^{\S}$ \\
\hline Insuficiente & $127(37,1)$ & $23,9(12,2-42,3)$ & & $2,8(0,37)$ & \\
\hline Adequado & $138(40,4)$ & $24,1(14,4-42,9)$ & & $2,8(0,34)$ & \\
\hline Excessivo & $77(22,5)$ & $24,6(14,1-49,5)$ & & $2,8(0,35)$ & \\
\hline Tipo de parto ${ }^{9}$ & & & $0,65^{\star}$ & & $0,35^{\ddagger}$ \\
\hline Normal & $170(45,9)$ & $24,0(10,7-51,6)$ & & $2,8(0,36)$ & \\
\hline Cesária & $200(54,1)$ & $24,0(7,3-49,5)$ & & $2,8(0,39)$ & \\
\hline $\begin{array}{l}\text { AME ou predominante aos } \\
\text { 4-6 meses }\end{array}$ & & & $<0,001^{*}$ & & $0,16^{\ddagger}$ \\
\hline Sim & $77(20,8)$ & $25,7(14,1-51,6)$ & & $2,9(0,38)$ & \\
\hline Não & $294(79,2)$ & $23,8(7,3-39,3)$ & & $2,8(0,38)$ & \\
\hline
\end{tabular}

VGP: velocidade de ganho de peso; VGC: velocidade de ganho de comprimento; AME: aleitamento materno exclusivo; Mín: mínimo; Máx: máximo; $\overline{\mathrm{X}}$ : média; DP: desvio-padrão. ${ }^{*}$ teste de Mann-Whitney. ${ }^{~}$ teste de Kruskal Wallis. ${ }^{~}$ teste t de Student; ${ }^{5}$ ANOVA. ${ }^{1} \mathrm{n}=369 .{ }^{2} \mathrm{n}=343 .{ }^{3} \mathrm{n}=345 .{ }^{4} \mathrm{n}=343 .{ }^{5} \mathrm{n}=356 .{ }^{6} \mathrm{n}=365 .{ }^{7} \mathrm{n}=362$. ${ }^{8} \mathrm{n}=342 .{ }^{9} \mathrm{n}=370$

variáveis estudadas. Com relação à prática do aleitamento materno, foi encontrado que $20,8 \%$ dos lactentes estavam em aleitamento materno exclusivo (AME) ou predominante nos primeiros quatro a seis meses de vida, além de apresentarem maior mediana de VGP quando comparados aos que recebiam fórmulas infantis ou outros leites ( $\mathrm{p}<0,001)$.

A maioria das mães $(72,8 \%)$ residia com o companheiro, fator que contribuiu para uma maior VGC nos primeiros meses de vida $(p<0,001)$. Os lactentes cujas mães realizaram maior número de consultas no pré-natal apresentaram maior VGC em comparação aos que realizaram menos que seis consultas $(\mathrm{p}=0,001)$. Adicionalmente, como apresentado na Tabela 2, os lactentes com maior comprimento ao nascer tiveram maior VGP $(\mathrm{p}=0,02)$ e menor VGC $(\mathrm{p}=0,01)$ nos primeiros quatro a seis meses de vida (Tabela 2 ).
Os resultados da análise múltipla estão apresentados na Tabela 3. Os lactentes que apresentaram menor VGP aos quatro a seis meses eram do sexo feminino $(p=0,01)$ e os que não estavam em AME ou predominante $(\mathrm{p}<0,001)$. Em relação à VGC, as menores velocidades foram apresentadas pelos lactentes cujas mães não viviam com o companheiro $(\mathrm{p}<0,001)$, filhos de mães que trabalhavam fora do $\operatorname{lar}(\mathrm{p}=0,03)$, aqueles cujas mães realizaram menos de seis consultas no pré-natal $(\mathrm{p}=0,03)$ e os com maior comprimento ao nascer. A avaliação do ajuste das análises de regressão linear mostrou adequação dos modelos.

\section{DISCUSSÃO}

A alta prevalência de excesso de peso e a menor prevalência de déficit observadas no presente estudo corroboram os resultados encontrados em outros trabalhos que avaliaram 
Tabela 2. Coeficiente de correlação para velocidades de ganho de peso e de comprimento de crianças nos primeiros meses de vida acompanhadas no PROLAC, segundo variáveis estudadas, Viçosa/MG, 2012

\begin{tabular}{|c|c|c|c|c|}
\hline \multirow{2}{*}{ Variáveis } & \multicolumn{2}{|c|}{ VGP (g/dia) } & \multicolumn{2}{|c|}{ VGC (cm/mês) } \\
\hline & $\mathbf{r}$ & Valor $\mathbf{p}^{*}$ & $\mathbf{r}$ & Valor $\mathbf{p}^{\dagger}$ \\
\hline Idade materna $(\text { anos })^{1}$ & 0,05 & 0,30 & 0,03 & 0,55 \\
\hline Altura materna $(\mathrm{m})^{2}$ & 0,03 & 0,45 & 0,04 & 0,37 \\
\hline IMC pré-gestacional materno $\left(\mathrm{kg} / \mathrm{m}^{2}\right)^{3}$ & 0,02 & 0,73 & 0,01 & 0,85 \\
\hline Peso ao nascer (g) & 0,04 & 0,36 & 0,01 & 0,78 \\
\hline Comprimento ao nascer $(\mathrm{cm})$ & 0,12 & 0,02 & 0,13 & 0,01 \\
\hline
\end{tabular}

VGP: velocidade de ganho de peso; VGC: velocidade de ganho de comprimento; g: grama; cm: centímetro; kg: quilograma; m: metro; r: coeficiente de correlação; IMC: Índice de Massa Corporal. ${ }^{*}$ Correlação de Spearman. ${ }^{\dagger}$ Correlação de Pearson. ${ }^{1} n=369 .{ }^{2} n=368 .{ }^{3} n=357$

Tabela 3. Modelos finais das análises de regressão linear múltipla para os fatores associados às velocidades de ganho de peso e de comprimento de crianças acompanhados no PROLAC, segundo fatores estudados, Viçosa/MG, 2012

\begin{tabular}{|c|c|c|c|c|c|c|c|}
\hline \multirow[t]{2}{*}{ Variáveis } & \multirow[t]{2}{*}{$\beta$} & $\begin{array}{c}\text { VGP } \\
\text { (g/dia) }\end{array}$ & \multirow[t]{2}{*}{ Valor $\mathbf{p}$} & \multirow[t]{2}{*}{ Variáveis } & \multirow[t]{2}{*}{$\beta$} & $\begin{array}{c}\text { VGC } \\
\text { (cm/mês) }\end{array}$ & \multirow[t]{2}{*}{ Valor $\mathbf{p}$} \\
\hline & & IC 95\% & & & & IC 95\% & \\
\hline Sexo & \multicolumn{7}{|c|}{ Estado civil } \\
\hline Masculino & - & - & \multirow[t]{2}{*}{0,01} & Com companheiro & - & - & \multirow[t]{2}{*}{$<0,001$} \\
\hline Feminino & $-0,05$ & $-0,10--0,01$ & & Sem companheiro & $-0,15$ & $-0,24--0,05$ & \\
\hline $\begin{array}{c}\text { AME ou } \\
\text { predominante }\end{array}$ & \multicolumn{7}{|c|}{ Trabalho materno } \\
\hline Sim & - & - & $<0,001$ & Não & - & - & 0,03 \\
\hline \multirow[t]{5}{*}{ Não } & $-0,10$ & $-0,15--0,04$ & & Sim & $-0,09$ & $-0,17--0,01$ & \\
\hline & \multicolumn{7}{|c|}{ No de consultas pré-natal } \\
\hline & & & & $<6$ & $-0,07$ & $-0,13-0,00$ & 0,03 \\
\hline & & & & $\geq 6$ & - & - & \\
\hline & & & & Comprimento ao nascer & $-0,03$ & $-0,06--0,01$ & $<0,001$ \\
\hline
\end{tabular}

VGP: velocidade de ganho de peso; VGC: velocidade de ganho de comprimento; IC: intervalo de confiança; $\beta$ : coeficiente de regressão linear; AME: aleitamento materno exclusivo

crianças nos primeiros meses de vida ${ }^{12,13}$. No estudo realizado por Gigante et al. ${ }^{13}$ em Pelotas/RS, ao compararem as coortes de nascimento de 1982 e de 1993, eles constataram aumento na prevalência de sobrepeso cerca de duas vezes maior nas crianças nascidas em 1993 quando comparadas com aquelas de idade semelhante nascidas em 1982. No entanto, houve diminuição na prevalência de déficit de estatura de quase 50,0\%. Na Pesquisa de Orçamentos Familiares realizada em 2008-2009, foi observada prevalência de $8,8 \%$ de baixo comprimento para idade em crianças menores de 1 ano, superior à observada no presente estudo $^{14}$. Um fator que pode ter contribuído para menor prevalência de déficit pôndero-estatural na presente amostra é que as crianças acompanhadas pelo PROLAC tinham as medidas antropométricas avaliadas a cada consulta, o que facilitava a detecção precoce de desvios nutricionais, os quais eram passíveis de controle ${ }^{3,4}$.

A menor VGP aos quatro a seis meses nas crianças do sexo feminino está de acordo com o encontrado no estudo longitudinal de Marques et al. ${ }^{15}$ em Belém/PA. No presente estudo, apesar de o sexo não ter sido um dos fatores explicativos para variação na VGC, foi observada maior média de ganho de comprimento entre os meninos nos três intervalos de idade avaliados.
Outra variável que explicou a variação na VGP foi a prática do aleitamento materno, pois as crianças que estavam em AME ou predominante apresentaram mediana de VGP de 25,7 g/dia, superior à observada para aquelas que não estavam em AME ou predominante $(23,8 \mathrm{~g} / \mathrm{dia})(\mathrm{p}<0,001)$. Na análise de regressão linear múltipla, após ajustes, o AME ou predominante se manteve como variável explicativa para variação da VGP aos quatro a seis meses. Outros estudos também demonstraram maior ganho de peso nos primeiros meses de vida entre lactentes em aleitamento materno quando comparados a aqueles alimentados com outros leites ${ }^{16,17}$. Resultados contrastantes foram verificados em trabalhos realizados em países em desenvolvimento, mostrando que, entre o terceiro e o sexto mês de vida, o ganho de peso de lactentes alimentados exclusivamente ao seio foi menor em relação aos que recebiam fórmulas ${ }^{18,19}$. A comparação dos resultados entre os estudos que avaliaram a influência da alimentação no crescimento nos primeiros meses de vida deve ser realizada com cautela, uma vez que são observadas diferenças entre as metodologias de obtenção da informação sobre a prática de aleitamento materno, bem como do referencial antropométrico $\operatorname{adotado}^{20,21}$. 
A prática do aleitamento materno não explicou variação da VGC entre as crianças do estudo, assim como observado por Spyrides et al. ${ }^{20}$. No entanto, outros estudos mostraram essa relação ${ }^{22,23}$. A prevalência de AME ou predominante aos quatro a seis meses observada no presente estudo foi de $20,8 \%$, semelhante à observada em lactentes de quatro a cinco meses participantes de um estudo de coorte realizado nas cinco regiões do Brasil ${ }^{22}$.

No modelo final de regressão linear múltipla, o estado civil materno manteve-se como variável associada para variações na VGC aos quatro a seis meses entre as crianças avaliadas. Ao analisar os dados do estudo "Demografic Health Surver", realizado em áreas de baixa renda em três países da América Latina e três do oeste africano, Desai ${ }^{24}$ identificou maior déficit de crescimento em filhos de mulheres que não tinham a presença de um companheiro. A presença do companheiro pode sugerir a possibilidade de maior obtenção de recursos financeiros para a família, com consequente melhora na qualidade de vida, fator determinante do crescimento infantil ${ }^{4}$. Outras variáveis socioeconômicas, como renda familiar e escolaridades materna e paterna, não foram estaticamente associadas à VGP e à VGC, no entanto há evidências na literatura que apontam a importância de se investigar tal associação $0^{4,25}$.

Foi encontrado que o trabalho materno fora do lar se associou à menor VGC aos quatro a seis meses de vida, resultado esse corroborado por outros autores ${ }^{26,27}$. Um dos fatores que pode justificar a menor VGC relacionada ao trabalho materno fora do lar é que, muitas vezes, essas mães interrompem o aleitamento materno e introduzem precocemente a alimentação complementar ${ }^{28}$. Sabe-se que a introdução precoce de alimentos complementares pode interferir negativamente na absorção de nutrientes e em sua biodisponibilidade, acarretando menor crescimento e aumento do risco de infecções respiratórias, alergias e diarreia nos primeiros meses de vida da criança ${ }^{29}$.

O número de consultas no pré-natal manteve a associação com a VGC nos primeiros quatro a seis meses de vida, mesmo após ajuste por outras variáveis estudadas. O adequado acompanhamento pré-natal permite a identificação de fatores que podem prejudicar o crescimento nos primeiros meses de vida e que são passíveis de controle, tais como o ganho de peso e a alimentação da gestante. Além disso, o maior número de consultas no pré-natal permite à gestante maior acesso a informações sobre os cuidados com a saúde e alimentação da criança, fatores diretamente relacionados ao crescimento infantil nos primeiros meses de vida ${ }^{30}$. Tem sido observado aumento da cobertura do atendimento pré-natal, com a realização de seis ou mais consultas pela maioria das gestantes, conforme preconizado pelo Ministério da Saúde. No entanto, verifica-se que a atenção pré-natal de qualidade ainda não atinge toda a parcela da população e que as gestantes com melhores condições de vida e maior escolaridade são as que mais consultam, evidenciando a desigualdade social ${ }^{29,30}$.

No presente estudo foi encontrado que o menor comprimento ao nascer esteve associado à maior VGC aos quatro a seis meses entre as crianças avaliadas, relação essa mantida no modelo múltiplo. No trabalho de Nandy et al. ${ }^{3}$, também foi observado o mesmo resultado, que pode ser atribuído ao crescimento compensatório que ocorre entre crianças que apresentam menor comprimento ao nascer.

Vale destacar que, pelo fato de nem todas as mães levarem os filhos às consultas mensalmente, não foi possível realizar a avaliação antropométrica dos lactentes a cada mês. Dessa forma, avaliaram-se os dados dos primeiros quatro a seis meses de vida, pois os valores diários de incremento pôndero-estatural são semelhantes nesse período².

Como limitação deste trabalho, vale ressaltar a forma de obtenção dos dados de peso e de comprimento ao nascer, ganho de peso e de comprimento aos quatro a seis meses de vida, aleitamento materno e outras variáveis analisadas, pois foram obtidos em registro de prontuários de atendimento. No entanto, o Programa de atendimento em questão é bem estruturado e possui protocolos estabelecidos, o que garante maior confiabilidade dos dados coletados.

\section{CONCLUSÃO}

Os resultados observados no presente estudo evidenciam a diversidade de fatores que podem estar associados ao crescimento nos primeiros meses de vida. Como a prática do AME ou predominante nos primeiros quatro a seis meses de vida foi associada ao maior ganho de peso, comparada à ingestão de outros leites, reforça-se a importância de ações efetivas voltadas ao "combate" das principais causas do desmame precoce, no intuito de reduzir a alta prevalência de abandono do aleitamento materno nos primeiros meses de vida e propiciar adequado desenvolvimento nesse período. Além disso, é de grande importância que as gestantes tenham acesso a um serviço de atenção pré-natal de qualidade e que realizem no mínimo seis consultas, como é preconizado, que foi considerado neste estudo como um fator que favoreceu a VGC no período avaliado.

É também necessário investigar durante a avaliação de lactentes variáveis relacionadas às condições de vida, como estado civil e trabalho materno, pois demonstraram estar associadas ao crescimento. A identificação precoce dos fatores relacionados ao crescimento infantil pode direcionar as ações dos profissionais saúde para que se alcance o crescimento adequado nessa fase. 


\section{REFERÊNCIAS}

1. Romani SAM, Lira PI. Determinant factors of infant growth. Rev Bras Saude Mater Infant. 2004;4(1):15-23. http://dx.doi.org/10.1590/S151938292004000100002

2. Augusto RA, Souza JM. Growth of children in exclusive breastfeeding in their first semester of life. Rev Bras Crescimento Desenvolv Hum. 2007;17(2):1-11.

3. Nandy S, Irving M, Gordon D, Subramanian SV, Smith GD. Poverty, child undernutrition and morbidity: new evidence from India. Bull World Health Organ. 2005;83(3):210-216.

4. Eickmann SH, Lima MC, Mota ME, Romani SA, Lira PI. Crescimento de nascidos a termo com peso baixo e adequado nos dois primeiros anos de vida. Rev Saude Publica. 2006;40(6):1073-81. http://dx.doi.org/10.1590/ S0034-89102006000700016.

5. World Health Organization. Growth velocity based on weight, length and head circumference: methods and development. Geneva: WHO; 2009.

6. Adair LS, Guilkey DK. Age-specific determinants of stunting in Filipino children. J Nutr. 1997;127(2):314-20.

7. Gonçalves SC, Louzada ML, Campagnolo PD, Vitolo MR. Weight gain rate and feeding practices of low-socioeconomic status infants. Rev Nutr. 2012;25(5):555-63.

8. Brasil. Ministério da Saúde. Vigilância Alimentar e Nutricional (SISVAN). Orientações básicas para a coleta, o processamento, a análise de dados e a informação em serviços de saúde. Brasília: Minstério da Saúde; 2004.

9. Brasil. Ministério da Saúde. Vigilância Alimentar e Nutricional (SISVAN). Classificação do Estado Nutricional. Brasília: Ministério da Saúde; 2009.

10. World Health Organization. Indicators for assessing breastfeeding practices. Geneva: WHO; 1991

11. Institute of Medicine. Weight gain during pregnancy: reexamining the guidelines. Washington: The National Academies Press; 2009

12. Matos SMA, Jesus SR, Saldiva SRDM, Prado MS, D'Innocenzo S, Assis AMO, et al. Velocidade de ganho de peso nos primeiros anos de vida e excesso de peso entre 5-11 anos de idade, Salvador, Bahia, Brasil. Cad Saude Publica. 2001;27(4):714-22. http://dx.doi.org/10.1590/S0102311X2011000400010

13. Gigante DP, Victora CG, Araújo CLP, Barros FC. Tendências no perfil nutricional das crianças nascidas em 1993 em Pelotas, Rio Grande do Sul, Brasil: análises longitudinais. Cad Saude Publica. 2003;19(Supl 1):S141-7. http://dx.doi.org/10.1590/S0102-311X2003000700015.

14. Brasil. Instituto Brasileiro de Geografia e Estatísticas. Pesquisa de orçamentos familiares 2008-2009: antropometria e estado nutricional de crianças, adolescentes e adultos no Brasil. Rio de Janeiro: IBGE; 2010.

15. Marques RF, Lopez FA, Braga JA. Growth of exclusively breastfed infants in the first 6 months of life. J Pediatr. 2004;80(2):99-105.

16. Hosseini SM, Maracy MR, Sarrafzade S, Kelishadi R. Child weight growth trajectory and its determinants in a sample of Iranian children from birth until 2 years of age. Int J Prev Med. 2014;5(3):348-355.

17. Spyrides MH, Struchiner CJ, Barbosa MT, Kac G. Effect of predominant breastfeeding duration on infant growth: a prospective study using nonlinear mixed effect models. J Pediatr. 2008;84(3):237-43. http://dx.doi.org/10.2223/ JPED.1797.

18. Naing KM, Co TT. Growth and milk intake of exclusively breast-fed Myanmar infants. Eur J Clin Nutr. 1991;45(4):203-7.

19. Díaz S, Herreros C, Aravena R, Casado ME, Reyes MV, Schiappacasse V. Breast-feeding duration and growth of fully breast-fed infants in a poor urban Chilean population. Am J Clin Nutr. 1995;62(2):371-6.

20. Spyrides MH, Struchiner CJ, Barbosa MT, Kac G. Amamentação e crescimento infantil: um estudo longitudinal em crianças do Rio de Janeiro, Brasil, 1999/2000. Cad Saude Publica. 2005;21(3):756-66. http://dx.doi. org/10.1590/S0102-311X2005000300009.

21. Ejlerskov KT, Christensen LB, Ritz C, Jensen SM, Molgaard C, Michaelsen KF. The impact of early growth patterns and infant feeding on body composition at 3 years of age. Br J Nutr. 2015;114(2):316-27. http://dx.doi. org/10.1017/S0007114515001427.

22. Longo GZ, Souza JM, Souza SB, Szarfarc SC. Crescimento de crianças até seis meses de idade, segundo categorias de aleitamento. Rev Bras Saude Mater Infant. 2005;5(1):109-18. http://dx.doi.org/10.1590/S151938292005000100014 .

23. César JA, Victora CG, Barros FC, Santos IS, Flores JA. Impact of breast feeding on admission for pneumonia during postneonatal period in Brazil: nested case-control study. BMJ. 1999;318(7194):1316-20. http://dx.doi. org/10.1136/bmj.318.7194.1316

24. Desai S. Children at risk: the role of family structure in Latin America and West Africa. Popul Dev Ver. 1992;18(4):689-717. http://dx.doi. org/10.2307/1973760.

25. Oliveira JS, Lira PIC, Carvalho AGC, Barros MFA, Lima MC. Fatores associados ao estado nutricional em crianças de creches públicas do município de Recife, PE, Brasil. Rev Bras Epidemiol. 2013;16(2):502-12. http://dx.doi.org/10.1590/S1415-790X2013000200024.

26. Lima M, Ismail S, Ashworth A, Morris SS. Influence of heavy agricultural work during pregnancy on birthweight in northeast Brazil. Int J Epidemiol. 1999;28(3):469-74. http://dx.doi.org/10.1093/ije/28.3.469.

27. Vannuchi MT, Thomson Z, Escuder MM, Tacla MT, Vezozzo KM, Castro LM, et al. Perfil do aleitamento materno em menores de um ano no Município de Londrina, Paraná. Rev Bras Saude Mater Infant. 2005;5(2):155-62. http:// dx.doi.org/10.1590/S1519-38292005000200003.

28. Demétrio F, Pinto EJ, Assis AMO. Fatores associados à interrupção precoce do aleitamento materno: um estudo de coorte de nascimento em dois municípios do Recôncavo da Bahia, Brasil. Cad Saude Publica. 2012;28(4):641-54. http://dx.doi.org/10.1590/S0102-311X2012000400004.

29. Allen LH, Gillespie SR. What works? A review of the efficacy and effectiveness of nutrition interventions. Geneva: Asian Development Bank; 2001.

30. Barros FC, Victora CG, Barros AJ, Santos IS, Albernaz E, Matijasevich A, et al. The challenge of reducing neonatal mortality in middle-income countries: findings from three Brazilian birth cohorts in 1982, 1993, and 2004. Lancet. 2005;365(9462):847-54. http://dx.doi.org/10.1016/S01406736(05)71042-4. 